\title{
Value of single acid-fast bacilli sputum smears in the diagnosis of tuberculosis in HIV-positive subjects
}

Tuberculosis (TB) is a major public health concern, particularly in third-world nations, where the prevalence is reportedly as high as $40 \%$ (Bhargava et al., 2001). Being the most common opportunistic infection among HIV-positive subjects, TB has re-emerged as a global emergency especially in the Indian subcontinent (Kumarasamy et al., 1995). India reportedly has more TB cases than any other country in the world (Kumarasamy et al., 2003). HIV has a substantial influence on the incidence, clinical manifestations, treatment and disease outcome of TB. In addition, the dual epidemic is a cause for concern in countries where these two infections are prevalent in epidemic proportions (Ramachandran et al., 2003).

Prompt diagnosis of TB is crucial for the success of ensuing treatment in any community setting. Conceptually, most of the available standard laboratory and mycobacteriology guideline texts advocate at least three consecutive sputum specimens to detect acid-fast bacilli (AFB) and performing sputum culture on patients suspected to have the disease (Nelson et al., 1998). Considering the huge economic burden imposed on the HIV/AIDS community to avail of expensive antiretroviral drugs and other diagnostic tests, it may be unmanageable for the patients to provide three consecutive specimens. In such a scenario, the usefulness of a single AFB smear in TB diagnosis needs further evaluation. Therefore, we compared the value of microscopy of a single AFB smear in TB diagnosis with a radiometric culture method as the 'gold standard'.

We compared the results of a single AFB sputum smear with those from AFB culture from $121 \mathrm{HIV}$-positive patients suspected to have TB during a 4-year period from June 2002 to May 2006. Sputum specimens were collected and processed following the standard procedure recommended by the
Table 1. Analysis of single AFB sputum smears using the BACTEC 460 TB as the gold standard

\begin{tabular}{|lcc|}
\hline Variable & Estimate (\%) & 95\% confidence interval \\
\hline Sensitivity & 53.3 & $38-68 \%$ \\
Specificity & 89.5 & $79.7-95 \%$ \\
Positive predictive value & 68 & - \\
Negative predictive value & 82 & - \\
\hline
\end{tabular}

BACTEC 460TB (Becton Dickinson) operations manual. Briefly, after inoculating the treated specimens in BACTEC 12B vials (Becton Dickinson), an initial smear was prepared, stained by the Ziehl-Neelsen method and observed under oil immersion for AFB. The inoculated vials were incubated at $37{ }^{\circ} \mathrm{C}$ in the presence of $5-10 \% \mathrm{CO}_{2}$ and read in the BACTEC $460 \mathrm{~TB}$ following the manufacturer's instructions. Following primary isolation, the M. tuberculosis complex was identified by the $p$-nitro- $\alpha$-acetylamino- $\beta$-hydroxypropiophenone differentiation test. Analysis of data was carried out using the Statistical Package for Social Sciences (SPSS, Chicago, IL, USA) version 13.0. Particulars on the diagnostic test procedures used, viz. sensitivity, specificity, positive predictive value (PPV) and negative predictive value (NPV), were calculated to compare the AFB smear microscopy with culture.

The single AFB smear method showed a sensitivity of $53.3 \%$, specificity of $89.5 \%$, PPV of $68 \%$ and NPV of $82 \%$ (Table 1).
The increasing number of reports of mycobacteria other than tubercle (MOTT) as opportunistic pathogens in HIV disease and most reportedly culture-positive and smear-negative (Miguez-Burbano et al., 2006) suggested that we should investigate the influence of MOTT on the sensitivity and specificity of the single AFB smear method. When the MOTT-positive cases were excluded from the study population, interestingly, the sensitivity increased to $61.1 \%$. Furthermore, when the MOTT cases alone were considered and TB culture positives were excluded, the sensitivity decreased to $22.2 \%$ (Table 2).

Nonetheless, the specificity remained the same in both groups.

Rapid diagnosis of TB currently relies on AFB smear examination. As this cannot distinguish tubercle bacilli from MOTT, culture examination is therefore warranted. Furthermore, direct smear reportedly detects AFB only at concentrations of $\sim 10000$ bacilli $\mathrm{ml}^{-1}$ of the specimen. Conversely, as few as 100 bacilli $\mathrm{ml}^{-1}$ may be required for a positive
Table 2. Influence of mycobacteria other than tubercle (MOTT) on the sensitivity and specificity of single AFB sputum smears as compared with the BACTEC TB 460

\begin{tabular}{|lcclcc|}
\hline Variable & \multicolumn{2}{c}{ Excluding MOTT cases $(\boldsymbol{n}=\mathbf{1 1 2})$} & & \multicolumn{2}{c|}{ Excluding TB cases $(\boldsymbol{n}=\mathbf{8 5})$} \\
\cline { 2 - 3 } \cline { 5 - 6 } & Estimate (\%) & $\begin{array}{c}\mathbf{9 5} \% \text { confidence } \\
\text { interval }\end{array}$ & & Estimate $(\%)$ & $\begin{array}{c}\mathbf{9 5} \% \text { confidence } \\
\text { interval }\end{array}$ \\
\hline Sensitivity & 61.1 & $43.5-76.3 \%$ & & 22.2 & $3.9-59.8 \%$ \\
Specificity & 89.5 & $79.8-95 \%$ & & 89.5 & $79.8-95 \%$ \\
\hline
\end{tabular}


culture (Kant, 2001). It is widely known that TB transmission occurs before the level of bacilli reaches $10^{4} \mathrm{ml}^{-1}$ in the sputum, and during this period, the person continues to transmit the bacilli. The diagnostic value of sputum microscopy is reportedly eroded owing to the increasing number of HIV-related smear-negative pulmonary TB cases (Kant, 2001; Valadas et al., 2003) and the influence of MOTT, which substantially reduces the sensitivity of the single AFB smear method. Our study has shown that the AFB smear method has relatively lower sensitivity and specificity than the gold standard radiometric culture. However, three consecutive specimens may be required in prospective studies to improve the validity of the method. Hence we conclude that it may be necessary to choose AFB culture to diagnose TB in HIV-positive patients despite the high cost and time involved.

\section{Ramachandran Vignesh,} Pachamuthu Balakrishnan,
Esaki Muthu Shankar, Kailapuri Gangatharan Murugavel, Settu Hanas, Anitha Jebaraj Cecelia, Suniti Solomon and Nagalingeswaran Kumarasamy

YRG Centre for AIDS Research and Education, VHS Campus, Taramani, Chennai 600 113, India

Correspondence: Nagalingeswaran

Kumarasamy

(kumarasamy@yrgcare.org)

\section{References}

Bhargava, A., Jain, A. \& Agrawal, S. K. (2001). A comparison of liquid and solid culture media with radiometric system for detection of mycobacteria in clinical specimens. Indian $J$ Tuberc 48, 9-12.

Kant, L. (2001). Improving detection of infectious cases. Indian J Tuberc 48, 115-116.

Kumarasamy, N., Solomon, S., Paul, J., Vennila, R. \& Amalraj, A. R. (1995). Spectrum of opportunistic infections among AIDS patients in Tamil Nadu, India. Int J STD AIDS 6, 447-449.
Kumarasamy, N., Solomon, S., Flanigan, T. P., Hemalatha, R., Thyagarajan, S. P. \& Mayer, K. H. (2003). Natural history of human immunodeficiency virus disease in southern India. Clin Infect Dis 36, 79-85.

Miguez-Burbano, M. J., Flores, M., Ashkin, D., Rodriguez, A., Granada, A. M., Quintero, N. \& Pitchenik, A. (2006). Non-tuberculous mycobacteria disease as a cause of hospitalization in HIV infected subjects. Int $J$ Infect Dis 10, 47-55.

Nelson, S. M., Deike, M. A. \& Cartwright, C. P. (1998). Value of examining multiple sputum specimens in the diagnosis of pulmonary tuberculosis. J Clin Microbiol 36, 467-469.

Ramachandran, R., Datta, M., Subramani, R., Baskaran, G., Paramasivan, C. N. \&

Swaminathan, S. (2003). Seroprevalence of human immunodeficiency virus (HIV) infection among tuberculosis patients in Tamil Nadu. Indian J Med Res 118, 147-151.

Valadas, E., Hansched, T., Fernandes, M. L. \& Antunes, F. (2003). Smear microscopy to diagnose TB early and prevent further transmission in a population with a high prevalence of HIV infection. Clin Microbiol Infect 9, 1045-1047. 